\title{
El Proyecto Oviedo en Vassar College: una iniciativa de traducción colaborativa para Gonzalo Fernández de Oviedo y su Historia general y natural de las Indias
}

\section{The Oviedo Project at Vassar College: a collaborative translation initiative for Gonzalo Fernández de Oviedo's Historia general y natural de las Indias}

\author{
Nicolás Vivalda \\ Vassar College \\ ESTADOS UNIDOS \\ nivivalda@vassar.edu \\ [Hipogrifo, (issn: 2328-1308), 9.2, 2021, pp. 539-550] \\ Recibido: 23-01-2021 / Aceptado: 23-02-2021 \\ DOI: http://dx.doi.org/10.13035/H.2021.09.02.41
}

Resumen. El Proyecto Oviedo es una iniciativa digital colaborativa que apunta a producir la primera traducción completa de la Historia general y natural de las Indias de Gonzalo Fernández de Oviedo al inglés. Llevada a cabo por los mismos estudiantes de Vassar College y actualmente en las primeras fases de desarrollo, la iniciativa incluirá una versión anotada de la traducción completa de la Historia General, acompañada de materiales críticos e interpretativos adicionales. El concepto de traducción colaborativa requiere el constante intercambio de ideas entre los editores del proyecto y los estudiantes, divididos entre traductores y anotadores de la obra. El objetivo inicial de este esfuerzo académico es la compleción de la traducción al inglés para 2026, año del quinto centenario de la publicación de 
los primeros fragmentos del texto en el Sumario de la natural historia de Indias. Trabajando con un grupo de colaboradores de entre diez y quince estudiantes, el proyecto se propone revitalizar la lectura de Oviedo entre los investigadores que no necesariamente manejen el nivel de lengua necesario para acceder al texto original, a la par que descubrir o revisitar capítulos que, aunque ausentes en las traducciones del Sumario, todavía conservan una importante riqueza histórica, con potencial para futuros estudios coloniales, especialmente en el campo de nuevas tendencias académicas como los estudios bélicos, la ecocrítica y el estudio de animales.

Palabras clave. Gonzalo Fernández de Oviedo; Historia general y natural de las Indias; conquista del Nuevo Mundo; historia natural; traducción.

Abstract. The Oviedo Project is a collaborative digital initiative that aims to produce the first complete translation of Gonzalo Fernández de Oviedo's Historia general y natural de las Indias into English. Produced by Vassar College students and currently in the early stages of development, the initiative will result in the final publication of an annotated translation of the fifty books of the Historia general, as well as additional critical and interpretative materials. The concept of collaborative translation requires the constant exchange of ideas between the editors of the project and the students, divided between translators and annotators of the work. The preliminary aim of this academic effort is the completion of the English translation by 2026 , the $500^{\text {th }}$ anniversary of the Sumario de la natural historia de las Indias -the earliest iteration of the text- published in 1526. Working with an ever-replenishing group of collaborators of between ten and fifteen students, the project aims to revitalize the reading of Oviedo's work in researchers who do not necessarily have the level of Spanish language necessary to access the original text. In doing so, the project will contribute to the process of discovering or revisiting important chapters that, although absent in the translations of the Sumario, still contain an outstanding historical richness, with potential for future colonial studies, especially in the vein of new analytical trends, such as war studies, animal studies, and ecocriticism.

Keywords. Gonzalo Fernández de Oviedo; Historia general y natural de las Indias; Conquest of the New World; Natural History; Translation.

El Proyecto Oviedo nace en Vassar College por iniciativa de la profesora Lizabeth Paravisini-Gebert en la primavera de 2018 y responde, en gran medida a una conjunción de factores que hacen a la vida académica reciente de nuestra institución: una universidad de artes liberales ubicada en el valle del río Hudson. Como factores auspiciantes del proyecto, debo mencionar, en primer lugar, el crecimiento sostenido que el programa de estudios medio-ambientales ha experimentado en los últimos años de nuestra historia curricular. El programa multidisciplinario con especialización en estudios medio-ambientales fue el resultado de un esfuerzo conjunto que comenzó a tomar forma en el año 1995, a partir de una serie de iniciativas pedagógicas experimentales que incluyeron la enseñanza conjunta de clases, una serie de charlas y un seminario diseñado especialmente para profesores. Una conferencia 
en estudios medioambientales alojada en Vassar y auspiciada por el National Endowment for the Humanities se convirtió en el punto simbólico clave que contribuyó al nacimiento del nuevo programa de estudios, aprobado por el profesorado en diciembre de 1999 y certificado por el estado de Nueva York en los primeros meses del año $2000^{1}$.

En segundo lugar, debo remarcar un crecimiento en el número de cursos de literatura colonial que ofrece el departamento de estudios hispánicos, así como al menos tres promociones de estudiantes que han sabido combinar estudios medioambientales y estudios hispánicos como especialización y sub especialización de forma simultánea y alternativa en rango. Si menciono tan detalladamente el programa de estudios medioambientales como pieza de inspiración clave es por la persistente atmósfera de colaboración científico-humanista que ha sabido sembrar en el panorama académico de nuestra institución. El programa ha rotado su dirección entre las ciencias naturales, las ciencias sociales y las humanidades de manera igualitaria y en este florecimiento conjunto y simultáneo también se ha inspirado la Prof. ${ }^{\text {a }}$ Paravisini-Gebert para proponer un proyecto tan ambicioso y de tanta relevancia para la historia del desarrollo de las ciencias biológicas, geográficas y antropológicas del continente americano.

En los últimos años, especialmente en los departamentos de idiomas y de estudios medievales, hemos adoptado, además, una iniciativa denominada «going to the source» ('recurriendo a la fuente'), esto es, incentivar a los estudiantes a analizar fuentes primarias de conocimiento. La tarea, como era de esperar, requiere gran esfuerzo de parte de los profesores y supone un acompañamiento exhaustivo en términos de disponibilidad y horas de consulta. En el transcurso de estas experiencias, los estudiantes van tomando progresivo contacto con herramientas teóricas de análisis retórico-textual y también con nociones de instrucción paleográfica dependiendo del tipo de material del que se trate. La experiencia busca, además, que los alumnos no solo accedan al material de estudio a través de la bibliografía secundaria especializada, sino que sean capaces de analizarlo utilizando ojos noveles.

Un objetivo pedagógico anexo de este modelo sería estimular a los participantes de la clase a no sentirse abrumados por el cúmulo de bibliografía especializada que muchas veces acompaña a textos vinculados al mundo medieval o de la modernidad temprana, animándoles a brindar su propia perspectiva, su propio punto de análisis informado a fenómenos que pueden pasar por el campo histórico, literario o artístico. En un nivel de desarrollo ideal, el grupo editorial mantiene la aspiración de que el proyecto funcione como una especie de puente que permita reducir la distancia entre estos textos "poco accesibles" y nuevos enfoques académicos en torno a conceptos tan revisitados y contemporáneos como los de historia, raza, etnicidad, historia natural o historia medioambiental.

Desde el punto de vista puramente operativo, el proyecto tuvo, además, su empuje decisivo en un profundo cambio curricular que se llevó a cabo durante el año académico 2019-20 y que implicó la combinación de clases regulares con expe-

1. Para más detalles ver Jones, 2011.

HIPOGRIFO, 9.2, 2021 (pp. 539-550) 
riencias "intensivas" para estudiantes que deseen tomar estudios o desarrollar proyectos breves pero concentrados en un tema específico. Esos cursos intensivos², diseñados especialmente desde la cercanía intelectual con los alumnos (divididos en grupos más pequeños de los que constituyen una clase regular), son precisamente el vehículo que nos permite atrevernos a intentar la tarea hercúlea de traducir al inglés, por primera vez y en su totalidad, la Historia general y natural de las Indias, islas y tierra-firme del mar océano de Gonzalo Fernández de Oviedo.

En el caso específico del Proyecto Oviedo, el objetivo institucional de esta iniciativa de "ir a las fuentes" se ha ampliado entonces con un doble propósito para los estudiantes, pues no solo se trata de recurrir al texto primario en su edición del siglo XIX sino también de intentar traducirlo. La intención final es crear una nueva fuente en traducción donde otros académicos y/o estudiantes puedan abrevar con la asiduidad que una obra tan valiosa como la de Oviedo merecería en inglés. Como mencionaba en el párrafo anterior, la traducción completa es tarea poco menos que hercúlea porque se trata de los famosos cuatro volúmenes escritos entre 1524 y 1548.

¿Qué es entonces el Proyecto Oviedo? Pues una empresa colaborativa de traducción del texto completo de la Historia general a través de un modelo intensivo de talleres y consultas llevadas a cabo con tres de los profesores del Departamento de Estudios Hispánicos: la Prof. a Lizabeth Paravisini-Gebert, el Prof. Michael Aronna y quien escribe estas páginas. El objetivo último es lograr una traducción confiable, académica y fluida de la Historia general. El desarrollo de la traducción es en principio cronológico, pero no compulsivo, pues dentro del rango de los primeros volúmenes, los estudiantes han conservado la libertad de elegir aquellos capítulos del texto que más se acerquen a sus intereses. En este sentido, los participantes aprenden en el camino ciertos aspectos esenciales de traducción, además de conceptos ajustados acerca de cómo desarrollar una versión en inglés de una fuente originalmente producida en el siglo XVI.

El objetivo final del Proyecto Oviedo es tener entonces una traducción completa de la obra para el año 2026, quinto centenario de la publicación de la primera edición del Sumario (1526). La edición con la que estamos trabajando es la que pertenece a José Amador de los Ríos quien, entre 1851 y 1855, revisó una publicación en cuatro volúmenes, auspiciada por la Real Academia de Historia. La decisión de utilizar la edición de Amador de los Ríos como base para la traducción acelera nuestras posibilidades de avanzar más rápidamente en una iniciativa liderada por estudiantes sub-graduados de diferentes niveles de experiencia con la lengua española. Apostamos además a mantener el aparato de notas que el mismo crítico

2. Derivados del programa Vassar's Intensive Experiential Work Initiative (VIEW). 
cordobés diseñó para su edición, insertando algunas de nuestra propia factura³. Confiamos, en otras palabras, en la calidad que conserva la versión de Amador de los Ríos, así como también en su capacidad para sostener el rango de la palabra original de Oviedo 4 .

El piloto del programa se completó como una serie de estudios independientes y se llevó a cabo en el semestre de otoño de 2018, siendo recibido con mucho entusiasmo por los estudiantes. Para finales de la primavera de 2019 ya se habían traducido cuarenta y dos capítulos de la obra y para noviembre del mismo año el número alcanzaba ya los cien capítulos. Actualmente nos encontramos en un número cercano a los doscientos veinte capítulos, eso equivale a unas dos terceras partes del primer volumen. También nos encontramos en proceso de enriquecer este desafío con materiales críticos e ilustraciones contemporáneas al texto, con la expresa intención de invitar al campus de nuestra institución a especialistas en Oviedo que puedan ayudarnos a profundizar aun más el compromiso de los estudiantes con el proyecto ${ }^{5}$. Mientras preparamos una posible publicación física de los volúmenes, el grupo de profesores a cargo de la empresa decidió que la disponibilidad del material no podía quedar en suspenso, y para ello se ha creado una versátil página web (<pages.vassar.edu/oviedo/>) en la que puede navegarse la Historia general por libro y capítulo, indicándose también a qué estudiante corresponde la traducción primaria de cada uno de los textos.

3. Nuestra iniciativa contó en este punto con la valiosa colaboración de Eduardo Rodríguez Santiago, quién ofició de asistente de edición desde los comienzos del proyecto hasta julio de 2020. Por intermedio de The Ford Scholars Program at Vassar College, la estudiante Kendall Simmons también fue instrumental al desarrollo visual de la página web, encargándose de las ilustraciones y la puesta al día de los trabajos traducidos.

4. No queda descartado para una etapa posterior del proyecto el desarrollo de un seminario avanzado en el que estudiantes de último año pudiesen trabajar a partir de la edición príncipe, analizando en detalle las ilustraciones originales y reflexionando de manera histórica sobre el proceso editorial que permite traer a nueva vida a textos de la temprana Edad Moderna. Una clase como la descripta podría enfrentar a los estudiantes a cuestiones clave del trabajo editorial histórico, inspirando una apreciación creciente de tareas tan complejas como la modernización de grafías o la instauración de un esqueleto de notas sólido y coherente. La edición príncipe más cercana a nuestra institución se encuentra alojada en The Library Company de Filadelfia y sería perfectamente factible organizar una visita para que los estudiantes pudieran familiarizarse con la materialidad del volumen. La redacción original del proyecto propone, además, para los años 2024 y 2026, la organización de sendos simposios sobre la Historia general, eventos en los cuáles especialistas en la edición sevillana podrían ser invitados a colaborar de manera más cercana con estudiantes y profesores a cargo de la iniciativa.

5. Por ejemplo, la profesora Elizabeth Gansen, de Grand Valley State University, nos visitará en el otoño de 2021 para disertar sobre la estética renacentista en los grabados de Gonzalo Fernández de Oviedo. 

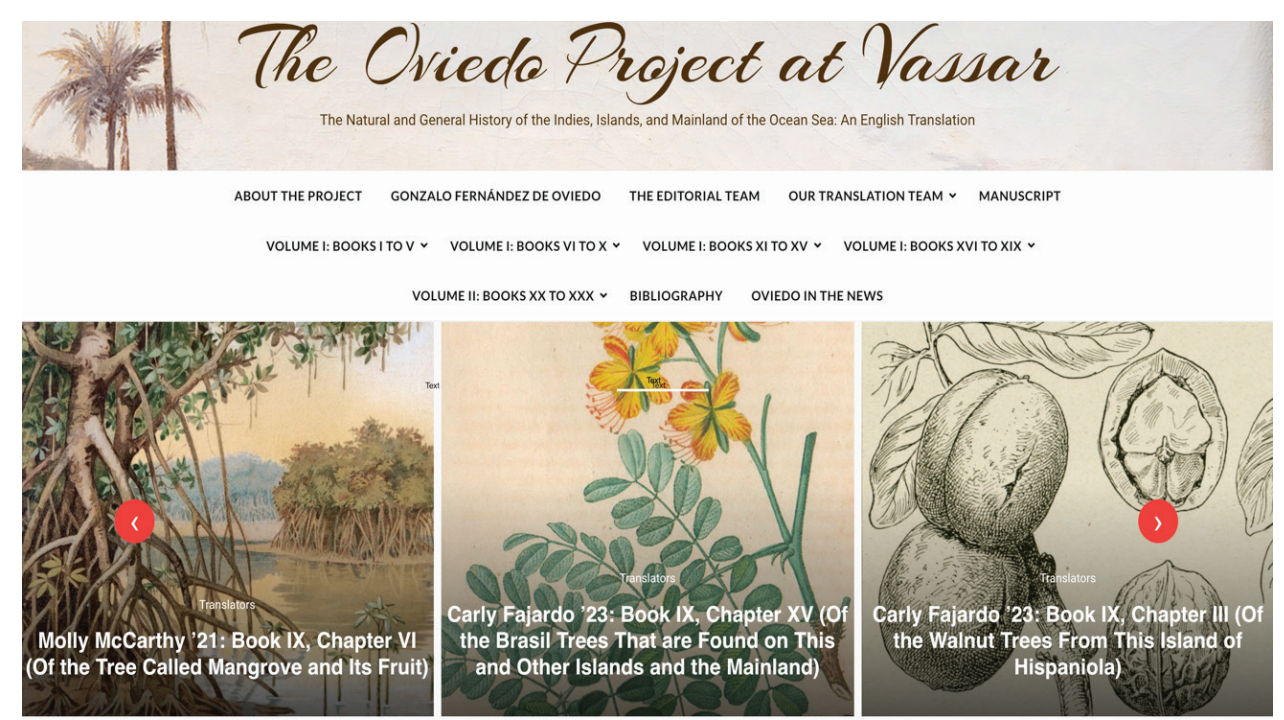

Fig. 1. La página de entrada del Proyecto Oviedo: <pages.vassar.edu/oviedo/>

En nuestras conversaciones preliminares, la carencia de una traducción completa del texto, se nos seguía ocurriendo como una falta importante para, por ejemplo, el desarrollo futuro de las denominadas humanidades medioambientales - campo de estudio que suele utilizar el inglés como lingua franca- que intenten concentrarse en la historia colonial temprana del territorio americano. En su punto de vista exclusivamente naturalista, el texto asume quizás una de sus dimensiones más preciadas, pues la descripción pionera de las diferentes especies mundonovistas nos lleva a comprender mejor el rápido desarrollo de la empresa colonizadora y la verdadera escala de sus efectos medioambientales, con la decadencia lenta -y posterior extinción- de muchas de esas mismas especies presentes en el libro. En Naturaleza e Imperio (2004), Jesús Carrillo habla precisamente de un texto de naturaleza orgánica, de evolución y crecimiento exponencial hasta la misma muerte del autor. Para Carrillo, la Historia general no puede ser analizada «como un obra cerrada e idéntica a sí misma, sino como un material que está constantemente edificándose y tomando forma sin adquirir ninguna definitiva o excluyente» ${ }^{6}$. La Historia general no deja de representar, en este sentido, un momento único en la presentación de la importancia política del discurso científico en América, pues su descripción sistemática de la naturaleza del Nuevo Mundo se ha transformado en una pieza clave en el análisis tanto de la historia de las ciencias naturales como del discurso colonial español. 
Recordemos brevemente que la estadía de Oviedo en tierras americanas se extendió por varias décadas, desde 1514 a 1557, y que la escritura de la Historia general ocupó una presencia casi continua en su agenda al menos hasta 1548. En el prólogo al mencionado libro de Carrillo, Anthony Pagden subraya precisamente este fenómeno de redacción extendida:

La Historia fue también el resultado de un proceso de laboriosa composición, y existen diversas versiones manuscritas del texto, tan lejos unas de otras como Madrid y California. A partir de ellas, Jesús Carrillo ha reconstruido trabajosamente la evolución "orgánica" -como él la denomina- cuyo crecimiento exponencial solo puso fin la muerte del autor en $1557^{7}$.

La ambición de registro fenoménico del Nuevo Mundo es, por supuesto, el punto clave que determina la extensión peculiar de la Historia. La definición de Kathleen Myers sigue siendo muy ajustada a este respecto:

Writing from the island of Hispaniola, the crossroads for the Spanish enterprise in the New World during the sixteenth century, Oviedo composed the most comprehensive history of the discovery, conquest, and colonization of the Americas from 1492 to 1547. Both a chronicle of the Spanish domination of America and a description of its flora, fauna, and indigenous peoples, the two-thousand-page general and natural history is the most authoritative text on the Americas from the first half of the sixteenth century ${ }^{8}$.

A medida que avanzamos en la traducción, la experiencia primaria de contacto con el texto se vuelve más evidente y un tanto abrumadora, pues por momentos parece que ningún fenómeno del Nuevo Mundo pudiera escapar de la atención de Oviedo ${ }^{9}$. Esa impresión - un poco agotadora en verdad- suele intensificarse en el lector no hispanohablante que se acerca por primera vez a esta extensa obra. Durante varios lapsos de la Historia, aunque Oviedo cambie la perspectiva, el acento de sus intereses descriptivos o la curva de sus transformaciones ideológicas, nunca ceja de transmitir una perspectiva pan-abarcante ${ }^{10}$ que, al mismo tiempo, fascina e intimida a los potenciales intereses de lectura ${ }^{11}$.

\section{Pagden, 2004, p. 15.}

8. Myers, 2007, p. 1

9. Jesús Carrillo habla del primer intento por producir un discurso histórico global sobre la empresa española en el Nuevo Mundo, primer punto que une la obra de Oviedo a la de Bartolomé de las Casas: «La Historia general y natural de las Indias (1535-1549) de Gonzalo Fernández de Oviedo es, junto con la Historia de Indias (1527-) de su contemporáneo y eterno contrincante, Bartolomé de las Casas, el primer proyecto orientado a producir un discurso histórico global sobre la empresa española en el Nuevo Mundo» (2004, p. 21).

10. El mismo Carrillo define a la Historia general también como una obra polifónica que, precisamente en su deseo de abarcar varios discursos y saberes, nunca termina de adquirir una forma definitiva o excluyente (2004, p. 26).

11. Según Kathleen Myers, la Historia general «reveals a man who worked constantly to navigate the complex politics and ambiguities of living and working in a colonial setting and in a period of rapid change in both Western history and historiographical practices» (2007, p. 25). 
Este afán por "describirlo todo" señala, indirectamente, el alcance de la idea que como cronista Oviedo tenía de sí mismo y de su tarea en territorio americano, un concepto construido en torno a una serie de cualidades innegociables de constante presencia en su obra: curiosidad profunda, extrema minuciosidad en la toma de notas, paciencia para escuchar testimonios diversos y, sobre todo, persistencia de indagación y constancia sin par entre los cronistas del Nuevo Mundo. Este altísimo nivel de comprehensión también define a la Historia general y nos enfrenta al mayor obstáculo en nuestro proyecto, pues no hace sino sumar a la dificultad de una traducción completa de un texto tan extenso. El desafío es evidente, pues el mismo Oviedo establece para su historia un nivel de exhaustividad nunca visto en la narración de la empresa de colonización de los territorios americanos ${ }^{12}$.

Ha de destacarse que la perspectiva testimonial y la valoración de la experiencia directa ${ }^{13}$ de los fenómenos que observa en el nuevo continente también agregan su parte a la extensión infrecuente de la obra ${ }^{14}$. Oviedo lleva sus indagaciones a niveles extremadamente minuciosos para ofrecer una narración que considera una fuente de verdad histórica irrefutable ${ }^{15}$. El fiel de ese horizonte de veracidad se basa, como ya mencionamos, en la experiencia directa de las cosas -la importancia del "haber estado alli"16 - o, frente a la ausencia de un testimonio directo, en

12. Dice Amador de los Ríos en su estudio preliminar a la edición de 1851: «Pero si aparecía violenta la comparación entre la cultura del mundo antiguo y del nuevo mundo, no menos peregrino era el desusado espectáculo que iba a desplegar a sus ojos aquella rica y varia naturaleza, virgen todavía a la codicia de los hombres y a las especulaciones de la ciencia. Todo había de ser nuevo en Oviedo dentro de breves meses, excitando poderosamente su imaginación, despertando con mayor fuerza aquel indefinible deseo de examinarlo y anotarlo todo y avivando al par el propósito ya concebido en 1492 de escribir la historia de las Indias» (p. XXIII).

13. «... nunca pude sentir ni entender las cosas de las Indias hasta que las vine a ver, e entendí muy diferenciadamente lo que antes había oído» (1974, Quinquagena II, p. 301).

14. Siempre conviene revisar la cita inaugural del volumen I de la Historia general, donde Oviedo intenta diferenciarse de Plinio (a pesar de que ha abrevado consciente y consistentemente en su prosa) por el camino de la experiencia directa -y el sufrimiento extremo- de múltiples vicisitudes: «Todos estos libros están divididos según género y calidad de las materias por donde discurren; las cuales no he sacado de dos mil millares de volúmenes que haya leído, como en el lugar suso alegado Plinio escribe, en lo cual me parece que él dijo que leyó; y algunas cosas dice que él acrecentó [...] pero yo acumulé todo lo que aquí escribo de dos mil millones de trabajos y necesidades y peligros en veintidós años y más que ha que veo y experimento por mi persona estas cosas, sirviendo a Dios y a mi rey en estas Indias y habiendo ocho veces pasado el gran mar Océano» (vol. I, libro l, p. 6).

15. Por poner un ejemplo muy conocido, el enfrentamiento con los "cronistas a distancia" alcanzaría un claro cenit en su discusión con Pedro Mártir en torno a la existencia y descripción exacta de los "lagartos de Tierra Firme": «Si aquí me he alargado tanto, ha sido para desengañar a los lectores de la opinión de Pedro Mártir. Pero no es solo en lo que sus Décadas se apartan de lo cierto de estas cosas de Indias, porque Pedro Mártir no pudo, desde tan lejos, escribir estas cosas tan al propio como son e la materia lo requiere; e los que le informaron, o no lo supieron decir, o él no lo supo entender» (vol. I, libro XII, capítulo VII, p. 393)

16. Una característica que comparte aun con su enemigo retórico Bartolomé de Las Casas: «For Las Casas, Oviedo was always that "utterly vain trifler" (vanissimus hic nugator), that unashamed fabricator of "monstrous lies [...] from which he stupidly promises himself immortality" whose writings, because of their undoubted popularity, had done more, in Las Casas's view, to harm the peoples of America that any 
la referencia múltiple a varias fuentes que hayan presenciado de primera mano el fenómeno del cual se encuentre hablando ${ }^{17}$. Son esos aspectos, ligados a la prolija exhaustividad y múltiple referencia de voces autorizadas, los que han dificultado la traducción completa de su opera histórica magna ${ }^{18}$. Recordemos que, en términos de traducción, el best seller de Oviedo continúa siendo la segunda edición del Sumario de 1535, texto mucho más breve que a pocos años de su publicación se tradujo al latín, inglés, francés, alemán e italiano. Esas traducciones contribuyen a las quince ediciones del Sumario publicadas durante el siglo XVI, fenómeno que habría de transformar al libro en un clásico doble de la etnografía y la historia natural renacentista.

La promesa de recompensa académica, tanto para profesores como para estudiantes del Proyecto Oviedo, consiste entonces en la apertura de una fuente primaria fundamental de la historia latinoamericana hacia el mundo no hispano-parlante. Este grupo de potenciales investigadores ha dependido, hasta el momento, de las distintas traducciones del Sumario o de ciertos capítulos o fragmentos traducidos como anexos de libros académicos sobre el mismo Oviedo. El entusiasmo primordial de los estudiantes implicados pasa entonces por la divulgación de zonas de la obra de Oviedo no necesariamente disponibles para el investigador que no maneje el idioma español. Uno de nuestras aspiraciones primarias es abrir, divulgar el rango completo de la Historia general para así hacer evidentes aspectos menos aludidos de su riqueza histórica y, de esta manera, contribuir a la investigación futura del texto. Un ejemplo de capítulo no-traducido que resalta por su ausencia (e implicaciones en el ámbito de los estudios zoológicos tempranos sobre el Caribe y Latinoamérica) es el que trata de los lobos marinos (vol. I, libro XIII, capítulo 5), traducido por la estudiante Adele Birkenes y que condensa en sus páginas intereses lingüísticos, históricos y de historia natural temprana del Caribe.

¿Quiénes son entonces nuestros traductores? Como Oviedo teje en su obra una multiplicidad de intereses tan vasta, el proyecto cuenta con un nivel de atracción eminentemente multidisciplinario. Hasta el momento hemos contado con la colaboración de estudiantes de especializaciones variadas y, en muchos casos, coincidentes en destrezas e intereses. Con respecto a las dificultades que estamos enfrentando, debo mencionar, en primer lugar, la desarmonía que los capítulos de la Historia general presentan entre sí, pasando la prosa de Oviedo por capítulos breves que lidian muy sucintamente con las cualidades de un animal o una fruta o hierba específica hasta capítulos extensísimos que describen circunstancias his-

other. Both Oviedo and Las Casas, however, were in agreement on one point: that only those who had "been there" could possibly have any significant understanding of America and its inhabitants» (Pagden, 1993, p. 57)

17. «The method, if it can be so called, which he employs in the Historia general consists in the simple forensic appeal to the multiplicity of "eye-witnesses". Like any competent jurist, he demands to know details about how and where his informants saw or heard what they have told him» (Pagden, 1993, p. 68). 18. Kathleen Myers considera a la Historia general como uno de los primeros repositorios históricos de información del Nuevo Mundo: «Gonzalo Fernández de Oviedo conceived of his historiographical project as an open-ended process of receiving new information that required continual revision -revision of canonical histories and revision of his own previous accounts» (2007, p. 82). 
tóricas con extrema prolijidad y frecuente espíritu digresivo ${ }^{19}$. Esa variedad es, sin embargo, también una ventaja estratégica para el propósito inmediato de conseguir traductores, pues nuestro grupo incluye desde colaboradores en su último año académico, hasta hablantes nativos de primer año que se acercan a la traducción por tener intereses previos en biología, geografía o estudios medioambientales. Algunos de los estudiantes más experimentados con el idioma han traducido incluso libros completos, como una forma de ganar crédito extra y completar lo que consideramos una tesis de traducción en estudios hispánicos. El nivel de ayuda y revisión que requieren de los profesores es, evidentemente, muy diferente dependiendo de cada uno de los niveles de idioma y de la madurez académica expresada por nuestros participantes.

Podríamos también añadir que, desde el punto de vista estrictamente lingüísti$\mathrm{co}$, las dificultades que hemos ido encontrando en el camino no son demasiado diferentes de las que Nina Scott describe en su valiosa aportación al libro de Kathleen Myers titulado Fernández de Oviedo's Chronicle of America (2007). Recordemos que Nina Scott, experimentada traductora de textos hispanos alto modernos, llevó a cabo la traducción de seis capítulos completos y algunos fragmentos de la Historia general y remarcó que sus principales obstáculos estuvieron relacionados con la "travesía lingüística" que Oviedo incita en todos sus lectores ${ }^{20}$. Ese "campo lingüístico minado" tiene que ver con múltiples niveles que afectan la comprensión del texto, a saber, niveles sintácticos, semánticos y aun ortográficos de variada complejidad para el lector actual. La lista de dificultades es fácilmente dirimible para cualquier lector que se acerque mínimamente a la obra de Oviedo: oraciones interminables (alejamiento constante de la referencia), conjunciones frecuentemente omitidas, inestabilidad ortográfica, elevación de los pronombres posesivos y, sobre todo, la aparición de algunas palabras oscuras o muy poco frecuentes aun para los lectores cultos de la época21.

Otro interrogante que suele presentársenos frecuentemente es por qué mantener un sitio web del proyecto. Aunque nuestro objetivo último sea la publicación de la Historia general en un formato preferentemente material, la idea es que podamos mostrar nuestro trabajo a medida que va completándose, señalando el avance de lo que consideramos un proyecto humanista multidisciplinario. La ventaja de llevar

19. En su ya seminal estudio sobre la naturaleza del Nuevo Mundo, Antonello Gerbi realiza un gran esfuerzo por separar el "lastre" de las farragosas digresiones de la característica más original de Oviedo, «cierta lucidez minuciosa de sus descripciones de la historia natural» $(1978$, p. 78). En History of the Conquest of Mexico, Prescott sería más impiadoso con el estilo de Oviedo: «His thoughts find themselves a vent in tedious, interminable sentences, that may fill the reader with despair; and the thread of the narrative is broken by impertinent episodes that lead to nothing» (1936, volumen IV, capítulo 8, p. 411).

20. Scott, 2007, p. 143

21. Nina Scott relata una simpática pesquisa en torno al término marítimo gisola (binnacle), que le llevó por múltiples diccionarios e indagaciones, hasta encontrar con la traducción que más se acercaba a la imagen que Oviedo pretendía evocar (2007, pp. 144-145) 
a cabo este tipo de proyectos en una institución como Vassar College pasa por la posibilidad de trabajar en pequeños grupos con los estudiantes, colaborando los profesores de manera personalizada con ellos y línea a línea en el proceso de traducción.

Lo que intentamos con el desarrollo y mantenimiento del sitio web es abrir un grado de disponibilidad más sincrónico al trabajo realizado, no demorar el acceso a investigadores que puedan estar buscando la traducción de un capítulo en particular o de un episodio histórico descrito en un fragmento del texto que no haya sido traducido hasta ahora. Las denominadas humanidades digitales nos ofrecen, en este sentido, nuevas formas de pensar la colaboración y el concepto mismo de aprendizaje experiencial, aportando en el camino novedosas vías de accesibilidad y almacenamiento para un texto tan comprehensivo como el de la Historia general. La página web se encuentra actualizada hasta el verano de 2020 y refleja nuestro empeño porque no transcurran más de ocho semanas entre la recepción de la primera versión del traductor/a y las revisiones que nos permiten anexar los nuevos capítulos al sitio. Nuestra presencia en la red funciona, además, como una celebración del entusiasmo y el compromiso de nuestros alumnos no solo por ir a la fuente, sino por contribuir a crear un nuevo texto de referencia. El desafío es, en este punto, también fomentar un nivel de entusiasmo lúdico y recreativo por el proyecto promoviendo, como ya se ha señalado, un texto que de por sí cuenta con un alto nivel de atracción entre estudiantes de español, historia, biología, geografía, estudios latinoamericanos y estudios medioambientales.

\section{BiBLIOGRAFÍA}

Carrillo Castillo, Jesús María, Naturaleza e Imperio. La representación del mundo natural en la «Historia general y Natural de las Indias» de Gonzalo Fernández de Oviedo, Madrid, Fundación Carolina, 2004.

Fernández de Oviedo y Valdés, Gonzalo, Historia general y natural de las Indias, islas y tierra-firme del mar océano, ed. José Amador de los Ríos, Madrid, Real Academia de Historia, 1851-1855, 4 vols.

Fernández de Oviedo y Valdés, Gonzalo, Las memorias de Gonzalo Fernández de Oviedo, ed. Juan Bautista Avalle-Arce, Chapel Hill, North Carolina Studies in Romance Languages and Literatures, 1974.

Gerbi, Antonello, La naturaleza de las Indias Nuevas, México, Fondo de Cultura Económica, 1978.

Jones, Elizabeth, «The History of Environmental Studies at Vassar College» (con contribuciones de H. Daniel Peck), en la página web Vassar750, marzo de 2011. 
Myers, Kathleen, Fernández de Oviedo Chronicle of America. A New History for a New World, translations by Nina Scott, Austin, University of Texas Press, 2007.

Pagden, Anthony, European Encounters with the New World, New Haven, Yale University Press, 1993

Pagden, Anthony, «Presentación», en Jesús María Carrillo Castillo, Naturaleza e Imperio. La representación del mundo natural en la «Historia general y natural de las Indias» de Gonzalo Fernández de Oviedo, Madrid, Fundación Carolina, 2004, pp. 11-16.

Paravisini-Gebert, Lizabeth, Aronna, Michael, y Vivalda, Nicolás (eds.), The Oviedo Project [página web].

Prescott, William Hickling, History of the Conquest of Mexico and History of the Conquest of Peru, New York, Modern Library, 1936.

Scott, Nina, «Nota del traductor» al «Appendix C», en Kathleen Myers, Fernández de Oviedo Chronicle of America. A New History for a New World, Austin, University of Texas Press, 2007, pp. 143-145. 